\title{
Laser management of phenytoin induced gingival enlargement in autistic child
}

\begin{abstract}
Drug-induced gingival enlargement is a frequent complication of phenytoin anticonvulsant therapy. In severe cases, gingival hyperplasia can cause marked cosmetic deformity and significant occlusal changes which severely restrict masticatory function. We describe a unique case of massive phenytoin-induced gingival overgrowth preventing the eruption of all teeth and subsequent dentofacial deformity. Carbon dioxide laser resection of the gingival enlargement, are discussed.
\end{abstract}

Keywords: gingival hyperplasia, gingival enlargement, co2 laser, phenytoin
Volume 8 Issue 7 - 2017

\section{Islam Kassem}

Department of Oral \& Maxillofacial Surgery, Qatar

Correspondence: Islam Kassem, Department of Oral \& Maxillofacial surgery, Qatar, Email ikassem@dr.com

Received: October 27, 2017| Published: November 29, 2017

\section{Introduction}

An increasing number of medications are associated with gingival enlargement. ${ }^{1,2}$ Drugs associated with gingival enlargement can be broadly divided into three categories: anticonvulsants, calcium channel blockers, and immunosuppressant. ${ }^{1}$ Phenytoin remains the drug of choice for treatment of grand mal, temporal lobe, and psychomotor seizures since it was first introduced in the 1930s. ${ }^{3-5}$ The first reported cases of phenytoin associated enlargement appeared more than 6 decades ago. Since then, other anticonvulsant agents have been introduced that have frequently been linked to clinically significant forms of gingival enlargement. ${ }^{1-10}$ The most effective treatment of drug-related gingival enlargement is withdrawal or substitution of medication. When this treatment approach is taken, as suggested by a case report, it may take from several weeks for resolution of gingival lesions. ${ }^{5}$ Unfortunately, not all patients respond to this mode of treatment, especially those with longstanding gingival lesions. ${ }^{6-8}$ The use of carbon dioxide lasers has shown some utility for reducing gingival enlargement, an approach which provides rapid postoperative homeostasis. ${ }^{1}$

\section{Case report}

3-year male child referred from the Maxillofacial Department, Alexandria University. The parent complains of unerption of all deciduous teeth together with dentofacial deformity. The child is autistic receiving anticonvulsive therapy (phenytoin). Intra oral examination shows tense fibrotic gingival, well developed ridge, averted upper lip, anterior open bite (Figure 1,2). CT scan examination revealed a generalized gingival enlargement, gingival tissue cover the whole teeth at all aspects. All teeth are present, with labioversion inclination of upper teeth, both alveolar bone \& basal bone is normal (Figure 3). The fibrous tissue band was resected using Carbon dioxide laser using KLS Martin Carbon dioxide Laser device with an infrared wavelength of $10,600 \mathrm{~nm}$. Safety precaution is applied. Teeth that are in close proximity to lased tissues should be protected. Common methods include draping moist gauze over the teeth (Figure 4). The new gingival margin was made by incising with the laser at 4 to $8 \mathrm{~W}$ in focused mode. Protection of the teeth is crucial and is achieved with either a thin freer elevator or a metal matrix band. When working in interproximal areas, an intermittent mode (20-millisecond pulses at 20 pulses per second) is done after the excess tissue was excised; the underlying hypertrophic tissue was sculpted appropriately. This process was performed in defocused mode at 6 to $10 \mathrm{~W}$.

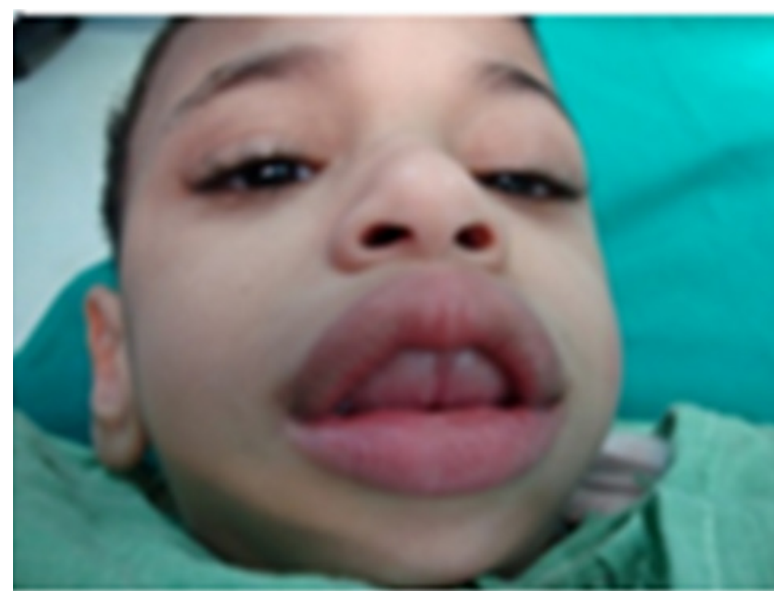

Figure I Clinical extra oral preoperative view.

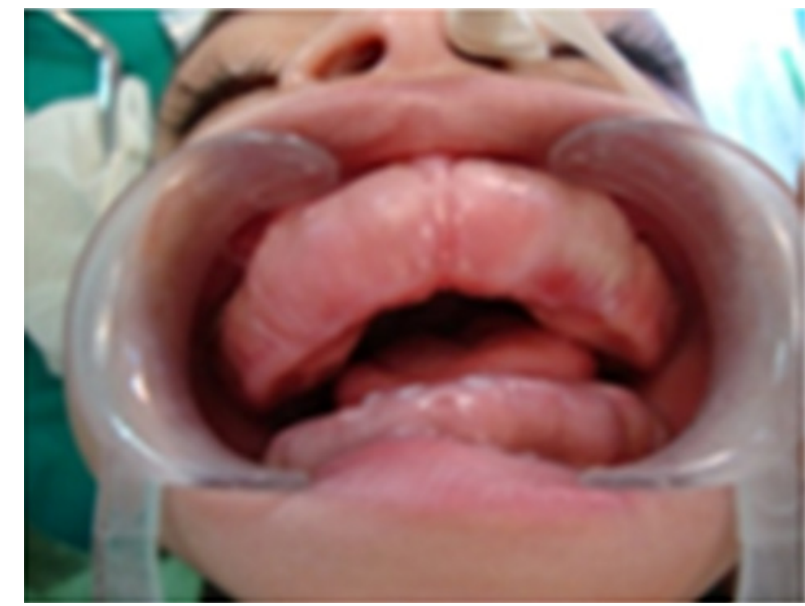

Figure 2 Clinical intraoral view. 


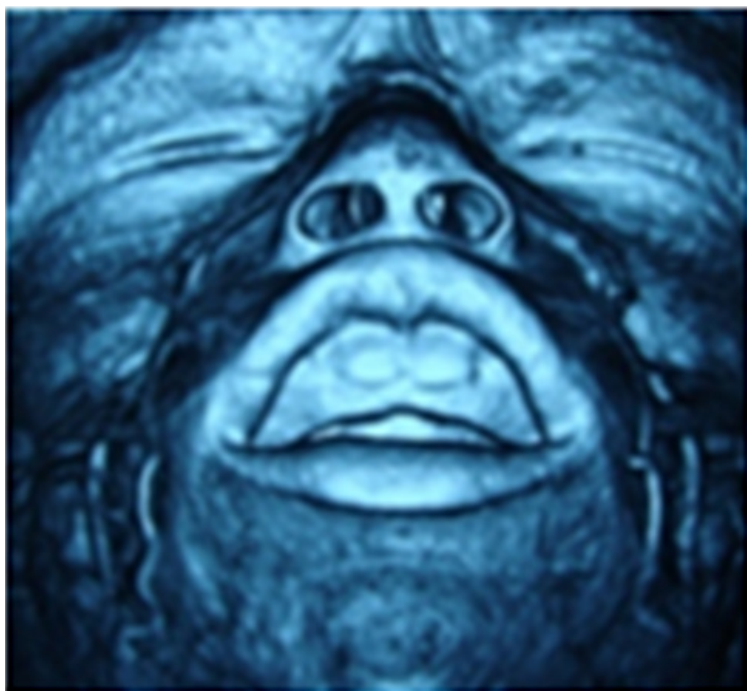

Figure 3 Radiographic preoperative view show fibrous band over deciduous teeth.

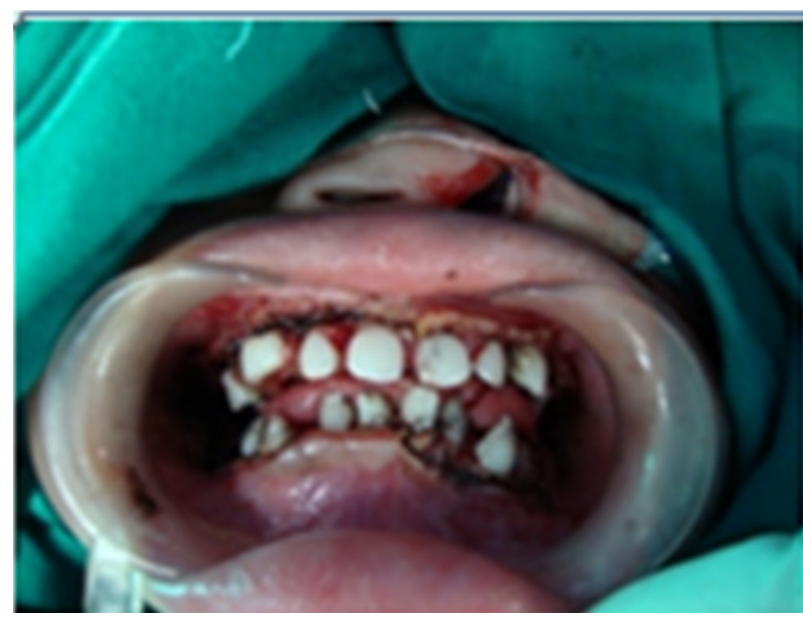

Figure 4 Immediate post operative view.

\section{Discussion}

The use of medications with the potential to contribute to the development of gingival overgrowth is likely increased in the years to come. Among the old and relatively new pharmacologic agents involved in gingival enlargement, overall, phenytoin still has the highest prevalence rate (approximately 50\%). Carbon dioxide lasers are now a standard of care for many oral and maxillofacial surgical procedures, as they are efficient for a variety of procedures. Despite the many advantages, there are also some disadvantages that must be weighed carefully before choosing this modality for patient treatment. As previously mentioned, healing from $\mathrm{CO}_{2}$ laser surgery is usually excellent, with decreased scarring and increased function ; however, the speed of healing usually is prolonged compared with other types of wounds. This delay in healing undoubtedly is due to the sealing of blood vessels and lymphatic's and the subsequent need for revascularization for healing. Laser management of drug induced gingival enlargement exhibit a solution for this health problem as its decrease bleeding, edema, pain \& recurrence provided good maintenance and oral hygiene.

\section{Acknowledgments}

None.

\section{Conflicts of interest}

None.

\section{Discussion}

The use of medications with the potential to contribute to the development of gingival overgrowth is likely increased in the years to come. Among the old and relatively new pharmacologic agents involved in gingival enlargement, overall, phenytoin still has the highest prevalence rate (approximately 50\%). Carbon dioxide lasers are now a standard of care for many oral and maxillofacial surgical procedures, as they are efficient for a variety of procedures. Despite the many advantages, there are also some disadvantages that must be weighed carefully before choosing this modality for patient treatment. As previously mentioned, healing from $\mathrm{CO}_{2}$ laser surgery is usually excellent, with decreased scarring and increased function ; however, the speed of healing usually is prolonged compared with other types of wounds. This delay in healing undoubtedly is due to the sealing of blood vessels and lymphatic's and the subsequent need for revascularization for healing. Laser management of drug induced gingival enlargement exhibit a solution for this health problem as its decrease bleeding, edema, pain \& recurrence provided good maintenance and oral hygiene.

\section{Funding}

None.

\section{Acknowledgments}

None.

\section{Conflicts of interest}

None.

\section{References}

1. Brunet L, Miranda J, Roset P, et al. Prevalence and risk of gingival enlargement in patients treated with anticonvulsant drugs. European Journal of Clinical Investigation. 2001;31(9):781-788.

2. Brunet L, Miranda J, Farré M, et al. Gingival enlargement induced by drugs. Drug Safety. 1996;15(3):219-231.

3. Kimball OP. The treatment of epilepsy with sodium diphenyl hydantoinate. JAMA. 1939;112(13):1244-1245.

4. Garnett WR, Louis EK, Henry TR, et al. Transitional polytherapy, tricks of the trade for monotherapy to monotherapy AED conversions. Curr Neuropharmacol. 2009;7(2):83-95.

5. Guncu GN, Cxaglayan F, Dincxel A, et al. Plasma and gingival crevicular fluid phenytoin concentrations as risk factors for gingival overgrowth. $J$ Periodontol. 2006;77(12):2005-2010.

6. Ando A, Aoki A, Watanabe H, et al. Bactericidal Effect of Erbium YAG Laser on Periodontopathic Bacteria. Lasers Surg Med. 1996;19(2):190 200 .

7. Fisher SE, Frame JW. The effects of the carbon dioxide surgical laser on oral tissues. Br J Oral Maxillofac Surg. 1984; 22(6): 414-425. 
8. Gold SI, Vilardi MA. Pulsed Laser Beam Effects on Gingiva. J Clin Periodontol. 1994;21(6):391-396.

9. Kanno CM, Oliveira JA, Garcia JF, et al. Effects of cyclosporine, phenytoin, and nifedipine on the synthesis and degradation of gingival collagen in tufted capuchin monkeys [Cebus apella]: histochemical and MMP-1 and -2 and collagen I gene expression analyses. J Periodontol. 2008;79(1):114-122.
10. Mcleod DE, Stoeckel D, Contreras J, et al. Severe postpartum gingival enlargement. J Periodontol. 2009;80(8):1365-1369. 